\title{
Bi-objective modeling approach for repairing multiple feature infrastructure systems
}

\author{
Nuno Sousa \\ INESC-Coimbra, Coimbra, Portugal \\ Department of Sciences and Technology, Universidade Aberta, Lisbon, Portugal \\ $\&$ \\ Luís Alçada-Almeida \\ INESC-Coimbra, Coimbra, Portugal \\ Faculty of Economics, University of Coimbra, Coimbra, Portugal \\ $\&$ \\ João Coutinho-Rodrigues* \\ INESC-Coimbra, Coimbra, Portugal \\ Department of Civil Engineering, Faculty of Sciences and Technology, University of Coimbra, Coimbra, Portugal
}

\begin{abstract}
A bi-objective decision aid model for planning long-term maintenance of infrastructure systems is presented, oriented to interventions on their constituent elements, with two upgrade levels possible for each element (partial/full repairs). The model aims at maximizing benefits and minimizing costs, and its novelty is taking into consideration, and combining, the system/element structure, volume discounts, and socio-economic factors. The model is tested with field data from 229 sidewalks (systems) and compared to two simpler repair policies, of allowing only partial or full repairs. Results show that the efficiency gains are greater in the lower mid-range budget region. The proposed modeling approach is an innovative tool to optimize cost/benefits for the various repair options and analyze the respective trade-offs.
\end{abstract}

\section{INTRODUCTION}

It is widely recognized that infrastructure systems require maintenance actions in order to mitigate deterioration and restore the system's condition (Pham et al., 1997; Zhang and Gao, 2012), or improve it. Factors such as environmental conditions, design characteristics, and utilization level (e.g. Durango and Madanat, 2002), as well as execution characteristics or maintenance actions (ISO 15686:2011), affect the service level provided by infrastructure systems to its users. Consequently, asset management tools have been introduced to help decision makers regarding how and when to repair/renew, estimate costs and determine the most effective maintenance strategy
(Golabi et al., 1982; Wang and Zaniewski, 1996; Capuruço and Tighe, 2006).

As reality evolves, the problem of determining optimal modalities of infrastructure asset management and maintenance changes accordingly. For instance, the phase of element design, construction scheduling and overall project management of infrastructures benefited from the appearance of object technology (see e.g. Yu and Adeli (1993); Chuang and Adeli (1993); Adeli and Yu (1995); Adeli and Kao (1996); Karim and Adeli (1999a, 1999b); Jiang and Adeli (2004)), whereas cost optimization of structures evolved through application of various emerging algorithms (Adeli and Wu (1998); Sarma and Adeli (1998); Sarma and Adeli (2000); Sarma and Adeli (2000); Kim and Adeli (2001); Sarma and Adeli (2001); Adeli and Sarma (2006)). After execution, the need for subsequent maintenance arises: Tak et al. (2013) claim there is a rising need for maintaining large-scale civil infrastructure systems in a sustainable way, due to climate change and taking into account budget constraints from the worldwide economic crisis. Michele and Daniela (2011) identified the following as factors that need be taken into account in the planning of management and maintenance actions: the progressive decrease in financial availabilities of public administrations; the higher citizens/users' attention to the supplied service quantity/quality; the interest in public health and safety, the population ageing and the consequent difficulties in accessing services; the obsolescence of many infrastructures realized during the town growths; and the increase in social/economic/environmental costs. As of late, the growing interdependencies among different infrastructure systems, and other positive and negative 
impacts associated to technological advances, have also become important in planning maintenance actions (Lee II et al., (2007); Nurre et al. (2012); González et al. (2016)).

However, at least at the municipal level, as recognized by Hegazy et al. (2012), management tools are primarily directed to support day-to-day management activities, and only an extremely small number of them offer limited support for long-term renewal planning, frequently not supporting some fundamental functions such as maintenance prioritization (Halfawy et al., 2005). The cost of applying maintenance and repair actions increases with the intensity of the action, with more intensive actions resulting in more significant improvements to a facility's condition. This bi-objective nature of the decision problem was recognized by Tak et al. (2013), who identified maintenance costs and infrastructure performance as key objectives to minimize/maximize respectively. The goal of this article is precisely to address this multiobjective problem.

The present research is thus motivated by this context of need for efficient, long-term maintenance/repair management tools, involving trade-offs between alternative capital investment plans and the corresponding levels of improvement. This article proposes a model to help decision makers planning for long-term repairs on infrastructure systems considering pressure from two sides: the need to maximize benefits coming from repairing the systems, and the need to minimize costs while doing so.

The setup considered for the application of the model is as follows. When facing maintenance of a particular class of infrastructure systems ('systems' for short) (e.g. municipal assets such as building facilities, roads, bridges, sewers, etc.), each member of this set of systems is inspected and analyzed relatively to its constituent elements (or parts, or objects), which may need to be repaired. The terms 'system' and 'element' can be understood as respectively 'alternatives' and 'criteria' on a multicriteria analysis context. The former lexicon is however preferred in this article for linguistic closeness to infrastructures terminology. The model considers that each element of each system can be left untouched or repaired to an endstate of either 'partially repaired' or 'fully repaired'. Partial repair restores the element to a pre-defined state, which is usually a minimum service level, but can be above that if the decision maker so wishes (elements that start out above partial level are treated in a slightly different manner - see model section for details). This partial level is subject to eventual practical engineering constraints, such as code of practice rules. Full repair brings the element to a "brand as new" state. End-states of 'full' and 'partial' repairs are also commonly referred in the literature for maintenance actions as 'perfect' and 'imperfect' restorations (Wang, 2002). Examples of systems/elements sets would be [1] buildings (systems) and their constitutive parts: foundations, roof, walls, windows, etc. (elements) (see e.g. Hegazy et al. (2012) for a full set of building elements); [2] highway segments (systems) and their constituents such as pavement, culverts, drainage system, berms, etc. (elements); [3] railroad tracks (systems) and their parts such as rails, ties, drainage system, etc. (elements) (Peng and Ouyang, 2014); [4] water/sewage networks branches (systems) and elements such as pipes, manholes, culverts, etc. In the authors experience, decision makers, especially at the municipal level, rarely consider multiple types of repairs on infrastructure, for lack of analytic methods to do so. The proposed modeling approach is one method which can cover that gap, enabling them to provide an overall better service.

The literature is abundant in models that help deciding whether to repair or replace faulty operating parts of machines (see e.g. Gao and Zhang, 2013), a problem which seems, at first sight, to share some similarities to the one presented here. However, the two are not equivalent problems for two reasons. First, repair/replace models usually address issues of operating parts wearing out due to use (e.g. mechanical parts or devices), and often use probabilistic techniques, like Markov chains, to model reality and evaluate which is the best course of action to undertake (Kijima et al., 1988; Love et al., 2000; Durango and Madanat, 2002; Dimitrakos and Kyriakidis, 2007). This study aims instead at planning long-term maintenance actions on large infrastructure systems, focusing on optimizing repair actions starting from the systems' present status, and not on finding the best strategy should parts fail sometime in the future. Second, in the present formulation the sub-problems to solve naturally synergize with each other because elements are not all independent: those belonging to a particular system share a structural relation that should be accounted for. For instance, it is expectable that fixing, say, a few trucks to a minimum road-worthy condition to be more beneficial than scattering equivalent repairs throughout a fleet without having none ready to operate in the end. The proposed approach reflects this structure of elements forming systems and system importance, whereas repair/replace models usually treat all sub-problems (i.e. parts to be repaired/replaced) in the same manner and independently, not taking into account possible relations among elements or element importance. A recent line of research which does take into account relations between infrastructures is that of optimizing post-disruption services restoration on interdependent networked infrastructure systems (Lee II et al., (2007); Nurre et al. (2012); González et al. (2016)). However, that problem (and it objective) is considerably different than the one considered in this article. A third difference lies in the cost part of the problem. Indeed, since the model addresses maintenance actions that can have a very large scale, it takes into account volume discounts that can be expected under real life market conditions for large orders. Although cost savings have been addressed in the literature (Furuya and Madanat, 2013), these were achieved by synergies from simultaneously working on several components, not by considering volume discounts.

The approach proposed in this study brings together many issues which are relevant in infrastructure systems maintenance: multiple repair levels, system/element structure, system importance, possible special relations among systems, and volume discounts. This makes it, as far as its authors can tell, different than other repair approaches in the literature. It is a new management tool for decision makers, which allows them to find cost-benefit optimized actions and analyze trade-offs. Its main application is longterm maintenance/repair planning; usually this is a context of pondered and timely planning, but one can envision that events causing sudden infrastructure downgrading (e.g. hurricanes, earthquakes) may cause the need for large-scale 
repairs. The model is to be used primarily by the contracting entity, as a means to search for the best course of action. The contractor itself can also use it to find the best application for the available budget.

\section{THE MODEL}

The model seeks to achieve the ideal combination of intervention actions in the constituent elements of each infrastructure system, so as to maximize the total benefits provided by the combined actions while minimizing their cost. Before presenting the model, its conceptual ideas and features are now summarized and some terminology is introduced:

a. Each element of each system starts off at a certain initial service level, usually reported from a field survey. The service level reflects the how well that element carries out its task and measures the benefits given by that element to the system's users. The element's final service level is determined by the optimization calculations, and can be equal to its initial level or an end-state of partial or full service level. If the initial service level of an element is below the level of a partial repair, two upgrade levels are available; otherwise see $(d)$. Below terms 'partially repaired' or 'fully repaired' are used as synonymous to endstate of partial/full service level.

$b$. In order to be used in the model, service level scales must be normalized to a $[0,1]$ interval, with 0 as 'no service' and 1 as 'fully operational'. In this scale, the partial service level is some threshold number $T$ between 0 and 1.

c. Elements with final service level equal to or greater than $T$ (but less than 1) are said to be partially repaired. Elements with final service level equal to 1 are said to be fully repaired.

$d$. Elements may have initial service level equal to or greater than $T$ (but less than 1), in which case they always end up partially repaired (in practice they are left untouched; the model treats this as partial repairs at zero cost - see subsection 2.1) or fully repaired. Similar considerations apply to elements that start out fully repaired, which sometimes happens.

$e$. Each system has an associated parameter called 'importance' that reflects its social, or economical, relevance. If the systems have a spatial dimension, this is where their size may enter the calculations, as well as intensity of use (usually 'importance $=$ size $\times$ intensity of use'). Element benefits are multiplied by this parameter.

$f$. Each type of element has an associated weight that reflects its importance to the whole of the system. Element benefits are multiplied by this weight.

$g$. The many elements under scrutiny form systems. To capture this structure, the model gives benefits bonuses to solutions that leave all elements of a system in an end-state of partial service level or better. Likewise, a benefit bonus (higher than the previous one) is given to systems that end up with all their elements at full service level.

$h$. If two systems that share some close relation among themselves are both given a bonus described in $(g)$ above, an extra synergy bonus may be awarded as on top of those. This applies mostly to spatial problems: a bonus for repairing contiguous systems, e.g. two sidewalks next to each other, which can thus provide a more continuous service to its users, but can be understood in a broader context. Say e.g. in a fleet of heavy-duty machines, attributing extra bonuses for solutions where an excavator and its carrying lorry are both put operational.

$i$. Costs for partial and full repairs are budgeted for each element of each system individually. These are just model parameters. As mentioned in $(d)$ above, if an element starts off with a service level above partial repair, its partial repair cost is set to zero.

$j$. Volume discounts are reflected in the cost structure for spending above two plateaus. Discounts are evaluated on a per-element basis, as there may be different suppliers and plateaus for each element. If a model solution dictates that the total (or cumulative) spending assigned to repairing a certain type of element goes above a certain plateau, the part of the spending that sits above that plateau gets a predefined discount.

The proposed model is formulated below as a mixedinteger linear problem (MILP) model, since this formulation allows for exact calculations and can thus serve as benchmark for variations of the model and/or other solving methods.

\subsection{Model formulation}

The mathematical model is formulated as follows. Consider $M$ systems, each with $N$ elements, and let index $i$ run through systems and index $j$ through elements, i.e. $i=$ $1, \ldots, M ; j=1, \ldots, N$. To keep the notation clean, all superscripts and subscripts in the text below are to be understood as labels (not exponents). Superscripts refer to cost or benefit levels, while subscripts refer to system/elements.

\section{Parameters:}

$S_{i j}$ : initial service level of element $j$ of system $i, S_{i j} \in$ $[0,1]$.

$T$ : service level threshold for partial repairs, $T \in] 0,1[$.

$I_{i}$ : socio-economical importance of system $i, I_{i}>0$.

$w_{j}$ : weight of element $j, \sum_{j} w_{j}=1$. Constant across all systems.

$B^{2}$ : benefit bonus if a system is at least partially repaired in all its elements (but not fully repaired in all of them), $B^{2}>0$.

$B^{3}$ : benefit bonus if a system is fully repaired in all its elements, $B^{3}>B^{2}$.

$B^{R}$ : benefit bonus if two specially related systems are at least partially repaired in all their elements, $B^{R}>0$.

$R_{i_{1} i_{2}}$ : binary parameter; 1 if systems $i_{1}, i_{2}$ are specially related, 0 otherwise, $i_{1}<i_{2}$.

$C_{i j}^{2}$ : cost of partially repairing element $j$ of system $i$, $C_{i j}^{2}=0 \forall_{i j}: S_{i j} \geq T, C_{i j}^{2}>0$ otherwise.

$C_{i j}^{3}$ : cost of fully repairing element $j$ of system $i, C_{i j}^{3}=$ $0 \forall_{i j}: S_{i j}=1, C_{i j}^{3}>C_{i j}^{2}$ otherwise.

$L_{j}^{1}, L_{j}^{2}$ : lower limit for respectively $1^{\text {st }} / 2^{\text {nd }}$ plateaus of cumulative spending on element $j, L_{j}^{2}>L_{j}^{1}>0$.

$D_{j}^{1}, D_{j}^{2}$ : discount for fraction of cumulative spending on element $j$ above $1^{\text {st }} / 2^{\text {nd }}$ plateau respectively. $1>D_{j}^{2}>$ $D_{j}^{1}>0$.

$K$ : auxiliary parameter, $K>\sum_{i j} C_{i j}^{3}$. 
Decision variables:

$x_{i j}^{1}$ : binary variable; 1 if element $j$ of system $i$ has a final service level below $T, 0$ otherwise.

$x_{i j}^{2}$ : binary variable; 1 if element $j$ of system $i$ is partially repaired, 0 otherwise.

$x_{i j}^{3}$ : binary variable; 1 if element $j$ of system $i$ is fully repaired, 0 otherwise.

\section{Dependent variables:}

$b_{i}^{2}$ : binary variable; 1 if all elements of system $i$ are at least partially repaired and at least one element is not fully repaired, 0 otherwise.

$b_{i}^{3}$ : binary variable; 1 if all elements of system $i$ are at fully repaired, 0 otherwise.

$r_{i_{1} i_{2}}$ : binary variable; 1 if systems $i_{1}, i_{2}$ are at least partially repaired in all their elements, 0 otherwise, $i_{1}<i_{2}$.

$c_{j}$ : cumulative spending for repairing element $j$ not considering discounts, $c_{j} \geq 0$.

$c_{j}^{0}:$ part of $c_{j}$ that has no discount, $c_{j}^{0} \geq 0$.

$c_{j}^{1}, c_{j}^{2}:$ part of $c_{j}$ above $1^{\text {st }} / 2^{\text {nd }}$ spending plateaus respectively, $c_{j}^{1} \geq 0, c_{j}^{2} \geq 0$.

$u_{j}^{1}, u_{j}^{2}$ : binary variable; 1 if $1^{\text {st }} / 2^{\text {nd }}$ discount plateau respectively is used when evaluating cumulative spending for repairing element $j, 0$ otherwise.

$$
\begin{aligned}
& \text { Model: } \\
& \max O_{1}=\sum_{i j: S_{i j}<T} I_{i} w_{j}\left(S_{i j} x_{i j}^{1}+T x_{i j}^{2}+1 x_{i j}^{3}\right) \\
& +\sum_{i j: T \leq S_{i j}<1} I_{i} w_{j}\left(S_{i j} x_{i j}^{2}+1 x_{i j}^{3}\right) \\
& +\sum_{i j: S_{i j}=1} I_{i} w_{j}\left(1 x_{i j}^{3}\right)+\sum_{i} I_{i} B^{2} b_{i}^{2} \\
& +\sum_{i} I_{i} B^{3} b_{i}^{3} \\
& +\sum_{i_{1}<i_{2}}^{i}\left(I_{i_{1}}+I_{i_{2}}\right) B^{R} R_{i_{1} i_{2}} r_{i_{1} i_{2}} \\
& \min O_{2}=\sum_{j} c_{j}^{0}+\sum_{j}^{i_{1}<i_{2}}\left(1-D_{j}^{1}\right) c_{j}^{1}+\sum_{j}\left(1-D_{j}^{2}\right) c_{j}^{2}
\end{aligned}
$$

$$
\begin{aligned}
& c_{j} \leq L_{j}^{1}+K u_{j}^{1} \quad ; \quad \forall_{j} \\
& c_{j}^{1} \leq c_{j}-L_{j}^{1}+K\left(1-u_{j}^{1}\right) \quad ; \quad \forall_{j} \\
& c_{j} \leq L_{j}^{2}+K u_{j}^{2} \quad ; \quad \forall_{j} \\
& c_{j}^{2} \leq c_{j}-L_{j}^{2}+K\left(1-u_{j}^{2}\right) \quad ; \quad \forall_{j} \\
& c_{j}^{0} \leq c_{j} \quad ; \quad \forall_{j} \\
& c_{j}^{2} \geq c_{j}-L_{j}^{2}-K\left(1-u_{j}^{2}\right) \quad ; \quad \forall_{j} \\
& c_{j}^{1} \leq L_{j}^{2}-L_{j}^{1}+K\left(1-u_{j}^{2}\right) \quad ; \quad \forall_{j} \\
& c_{j}^{1} \geq L_{j}^{2}-L_{j}^{1}-K\left(1-u_{j}^{2}\right) ; \quad \forall_{j} \\
& c_{j}^{0} \leq L_{j}^{1}+K\left(1-u_{j}^{1}\right) \quad ; \quad \forall_{j} \\
& c_{j}^{0} \geq L_{j}^{1}-K\left(1-u_{j}^{1}\right) \quad ; \quad \forall_{j}
\end{aligned}
$$

\section{Explanation:}

(1) Objective $\mathrm{O} 1$ receives three types of contribution. The first three terms of (1) represent raw benefits provided by systems to their users. The last of these is redundant and is only included for completeness. Next come bonuses to the benefit function coming from repairing all elements of a system at least partially, but not all fully (fourth term), and bonuses from fully repairing all elements of a system (fifth term). Finally, the last term (sixth) represents bonuses for repairing all elements of two specially related systems at least partially. (See section 2.2 for comments on how to set the bonuses values.) Terms 4-6 capture the system/elements structure of the problem and pair-wise relations between systems, making the model intrinsically different than planning repairs on a series of $i \times j$ disconnected elements.

(2) In objective $\mathrm{O} 2$ cumulative spending per element is distributed in three parts, or plateaus. First part has no discount, second part has a discount, and third part has a higher discount. This mimics volume discounts that can be expected under real life market conditions.

(3) These constraints define the possible course of action for each element of each system. Elements initially below $T$ can be either left untouched, partially or fully repaired (3a). Elements initially above, or at, $T$ can be partially or fully repaired $(3 \mathrm{~b})$, with partial repairs (at zero cost) being in this case just a formal definition, as in practice the element already starts out partially repaired. Finally, and in the same spirit, elements that start out fully repaired get automatically tagged for full repairs (3c) (again at zero cost).

(4) Constraints ( $4 \mathrm{~cd}$ ) force variable $b_{i}^{3}$ to be 1 if system $i$ is fully repaired in all its elements. Constraints ( $4 a b)$ force $b_{i}^{2}$ to be 1 if system $i$ is at least partially repaired in all its elements, but not fully repaired in all. Note that $b_{i}^{2}$ and $b_{i}^{3}$ cannot both be 1 simultaneously.

(5) These constraints activate $r_{i_{1} i_{2}}$ (i.e. set it to 1 ) when two systems end up with all their elements in an end-state of partial repairs or better. If the corresponding $R_{i_{1} i_{2}}$ is nonzero, i.e. if the systems are specially related, a benefit bonus is awarded.

(6) Constraints that define variable $c_{j}$ and relate it to the decision variables.

(7) If cumulative costs on element $j$ fall below a certain plateau limit, that plateau is not used, i.e. its corresponding $u_{j}$ becomes $0(7 \mathrm{ac})$ and costs assigned to it are also set to 0 (7bd).

(8) Constraints (8ac) ensure that $u_{j}$ is set to 1 when undiscounted cumulative spending on element $j$ surpasses the corresponding plateau lower limit. Constraints (8bd) 
assign spending above that limit to the corresponding plateau. Constraint (8e) is the equivalent of (8bd) for the spending part that has no discounts. Constraint (8f) ensures, together with (8d), that spending passed to the last plateau is exactly what remains to be distributed.

(9) Constraints (9ab) make sure that before moving to the next plateau, spending assigned to the previous one exhausts it. Constraints (9cd) are basically $(9 \mathrm{ab})$ for the cost part without discounts. For instance, if $u_{j}^{1}=1$, then $(9 \mathrm{~cd})$ enforce $c_{j}^{0}=L_{j}^{1}$ for any cumulative cost (since $8 \mathrm{a}$ only forces cumulative cost to stay below $L_{j}^{1}$ if $u_{j}^{1}=0$ ). Likewise for $(9 \mathrm{ab})$ if $u_{j}^{2}=1$, which enforces $c_{j}^{1}=L_{j}^{2}-L_{j}^{1}$ for any cumulative cost (since $8 \mathrm{c}$ only forces cumulative cost to stay below $L_{j}^{2}$ if $u_{j}^{2}=0$ ).

Note that constraints involving dependent variables keep the model linear, and thus exactly solvable. This completes the model presentation. The set of constraints was presented in an exhaustive way for clarity.

\subsection{Comments}

Below some further comments on how to parametrize and adjust the model to specific contexts are given.

Setting $B^{2}$ to, e.g. 0.20 , means that repairing the system to partial status makes it $20 \%$ better, as compared to just summing up benefits of individual elements, reflecting the fact that those elements coordinate properly. The system can thus carry out its task as intended, without shortcomings due to underperforming elements. Similarly, $B^{3}=0.40$ means that an impeccably repaired system is $40 \%$ better than the sum of individual benefits. It is up to the decision maker to judge what the plus-value of partially/fully repaired systems is, in terms of service quality. In an abstract context, the authors see $B^{2}=0.50$ and $B^{3}=1.0$ as sensible maximum values for these parameters (and $B^{R}=0.50$ maximum). Setting these ' $B$ bonuses' values is admittedly a subjective task. On the other hand, it may also be a plus-value since it allows different values for different infrastructures/contexts. Also, the decision maker can experiment with values until she finds a set she is comfortable with. Surveying various experts and averaging the result is yet another way to set these, and other, (potentially subjective) parameters, such as social-economic importance. Element weights may also require judgement, but in some cases codes of law fix these (see e.g. Natividade-Jesus et al. (2013), table 1).

Systems containing some elements that wear out fast can be approached by splitting the set of elements into two. Treat the fast wearing elements by e.g. one of the repair/replace methodologies cited in the introduction, and the remaining ones by the proposed modeling approach.

An interesting feature of the model is that it can also be used to plan for construction of new systems. Setting all $S_{i j}=0$ (system inexistent) and applying an extra constraint $x_{i j}^{1}=0$ (forces a construction action to be taken) will yield back optimized plans for the service level of each element of the new systems.

\section{CASE STUDY}

\subsection{Data and results}

The model was tested with field data from 229 sidewalks (systems) of the city of Coimbra, Portugal. Following the sidewalk performance evaluation methodology published in the Research Report of INESC-Coimbra available on http://www.uc.pt/en/org/inescc/res reports docs/RR 0420 $\underline{15}$, eight sidewalk elements were considered, namely width, pavement comfort, conservation status, accessibilities, safety from traffic, street lightning, obstacle density, and walking environment. The sidewalks in this study focused on three neighborhoods; two recent and an older one (the city's historical center), adding up to a length of $24.6 \mathrm{~km}$.

A brief summary of element data (service levels, costs) is presented in appendix A. Further details on data and its collecting can be found on the above-mentioned Research Report. In the description below, the cost structure (individual repair costs, plateau limits and discounts) was budgeted by a national civil engineering contractor. Costs include eventual demolitions needed and wages.

Model parameters were calibrated as follows. Service level threshold for partial repairs was set to $T=0.65$. This value was chosen over the more natural-looking $T=0.50$ because many sidewalks were in reasonable condition. Raising the threshold provided a wider range for the decision variables. Social importance was defined as sidewalk length multiplied by an integer factor of 1 to 6 , according to the sidewalk's HCM pedestrian density A to F score (TRB, 2000) $(\mathrm{A}=1)$. Element weights focused on comfort of use and were, by order of appearance in the first paragraph of this section, $w_{i}=$ $(0.05,0.15,0.35,0.10,0.05,0.05,0.10,0.15)$. Bonuses for system partial/full repairs were set to an extra $40 \% / 100 \%$ amount over raw benefits, i.e. $B^{2}=0.40$ and $B^{3}=1.0$. A total of 210 sidewalks next to each other (special relations) were identified and contiguity bonuses of $10 \%$ over raw benefits $\left(B^{R}=0.10\right)$ were set. Finally, plateau lower limits were set on a per-element basis (approximately 50\%/75\% total spending for the respective element) and discounts to $10 \% / 20 \%$ over $1^{\text {st }} / 2^{\text {nd }}$ limits respectively.

With the chosen parameterization, benefits ranged from 23,000 to 88,000 , where the lower/higher number represents respectively runtime/full repairs benefits, bonuses included. Cumulative solution costs ranged from 0 to 5,965,000 EUR after discounts $(6,647,000$ EUR raw costs - roughly $10 \%$ discount). The case study would thus require an investment just shy of 6 million EUR to quadruple benefits to the population.

Calculations were performed using IBM ILOG CPLEX v12.6.3 solver, running on a MacMini with Intel i7 3720QM quad-core@2.60 GHz. A large subset of the Pareto front for the problem was derived using the $\varepsilon$ constraint method (Cohon, 1978). Maximum benefit was constrained to range from 23,000 to 88,000 with intervals of 200 benefit units, and 326 runs were carried out for cost minimization. Infinitesimal weights of $10^{-5}$ were given to the constrained objective after normalization to ensure identification of a non-dominated solution (Ehrgott, 2005; Coutinho-Rodrigues et al., 2012A). CPU times ranged from 1 to 100 seconds per run, for a cumulative time of around 3 
hours and an average run time of $33.4 \mathrm{sec}$. This yielded the non-dominated solutions presented in Figure 1 below. In it the model's Pareto front is labeled as 'mixed recovery policy'. Although this may not be the whole front (there may exist more non-dominated solutions inside each interval of 200 units of benefit), it provides nevertheless an impression of it with enough resolution for trade-off analysis.

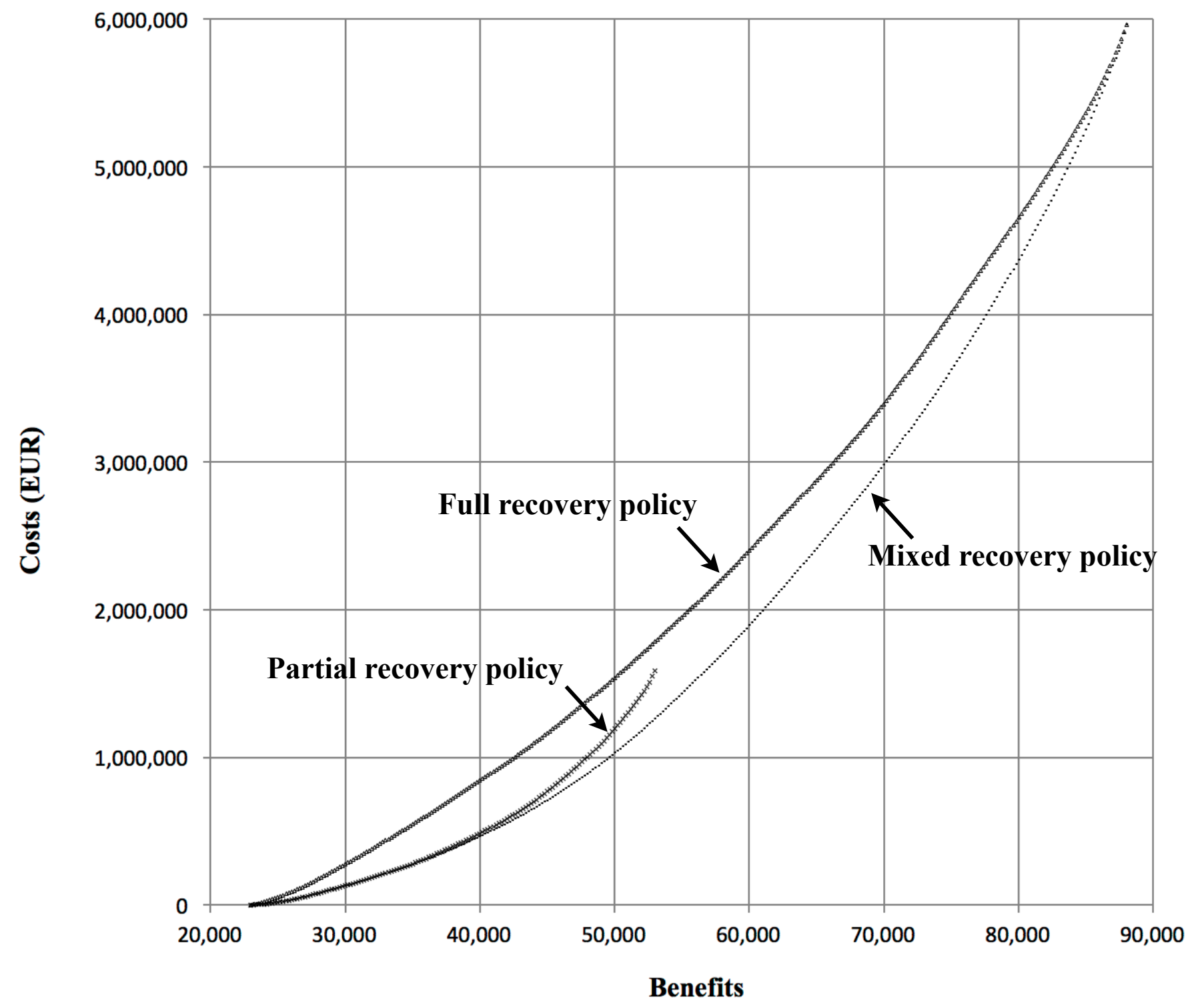

Figure 1 Case study: Pareto front for three repair policies

The front shows that the cost-benefit ratio is best at low costs, which was to be expected, as the model naturally selects the actions with best (lower) cost-benefit ratio first, and tends to spend its scarce money supply in reaching the systems' partial repairs bonuses (more on this in the discussion section). The cost-benefit ratio then gradually deteriorates, as repairs start to get more and more expensive. Differences in the cost-benefit ratio cause the front to exhibit a 'belly-shape'. This is a general feature, which is proportional to the standard deviation of the costbenefit ratio.

Having two upgrade levels gives flexibility in choosing the most appropriate repair type. In order to verify how large this advantage can be, the approach was compared to two other repair policies which the modeling approach allows to implement, of allowing only full or partial repairs. To implement the 'full recovery policy', the model was re- run with the additional constraints $x_{i j}^{2}=0 ; \forall_{i j}: S_{i j}<T$. For the 'partial recovery policy', the additional constraints were instead $x_{i j}^{3}=0 ; \forall_{i j}: S_{i j}<1$. Below terms 'partial/full recovery policy' refer to the model, run with the respective additional constraints, and 'mixed recovery policy' to the model in its native form; 'the model' for short.

The results of the partial/full recovery policy runs are also shown in Figure 1, under the respective label. Solutions of partial and full recovery policies are, by construction, a dominated subset of the mixed recovery policy, as the figure confirms. Note that the partial recovery policy front ends at around 53,000 benefits. This is the most benefits achievable from partial repairs only.

Figure 1 also shows that the partial recovery policy, a natural drive of decision makers, is similar in performance to the mixed recovery policy for low budget. However, as the budget increases, the mixed recovery policy becomes 
more and more efficient, in the sense that higher benefits can be achieved with the same spending. At benefit levels of 43,000 to 53,000 , the partial recovery policy requires $7 \%$ more costs $(+39,000$ EUR) to reach the same level of benefit, going up to $26 \%(+328,000$ EUR $)$ at the point where the partial front ends. It is at this 'lower mid-range' budget level that the model is most efficient.

To help understanding what the added benefits physically translate to, take a budget of 1,500,000 EUR as an example. For this amount of spending, the mixed recovery policy outperforms the partial recovery policy by 3200 benefit units. Referring to equation (1), and assuming e.g. all elements at $S_{i j}=0.25$, the 3200 extra benefits are equivalent to improvements of 0.25 to 0.65 on sidewalks whose total socio-economic importance is

$$
\begin{gathered}
B=I\left[(T-0.25)+B^{2}\right] \Leftrightarrow I=\frac{3200}{(0.65-0.25+0.40)} \\
=4000
\end{gathered}
$$

or, considering instead improvements from 0.25 to 1 ,

$$
B=I\left[(1-0.25)+B^{3}\right] \Leftrightarrow I=\frac{3200}{(1-0.25+1)} \approx 1830
$$

Since sidewalk importance is equal to its length times HCM pedestrian level, this means that with 1,500,000 EUR the mixed recovery policy can, as compared to the partial recovery policy, achieve extra benefits that are equivalent to partially repairing an extra $4000 \mathrm{~m}$ of badly damaged $\left(S_{i j}=0.25\right)$ HCM A-level sidewalk or fully repairing 1830 $\mathrm{m}$ of the same sidewalk (respectively $2000 \mathrm{~m} / 915 \mathrm{~m}$ of HCM B-level sidewalk, etc.) (recall that total sidewalk length is $24.6 \mathrm{~km}$ ). And it achieves this by optimizing the type of repair to perform on each element of each sidewalk.

Note that benefit units can always be translated into equivalent physical reality in the above manner.

\subsection{Solutions minimizing distances to the ideal}

As seen, the mixed recovery policy dominates the other policies more clearly at the lower mid-range budget region. If the decision maker's budget is large enough to reach this region, choosing a particular solution for field implementation can be further helped by calculating distances to the ideal, as measured by an $L_{p}$-norm (Coutinho-Rodrigues et al., 2012A). The $L_{p}$ distance indicates how close a solution is to the ideal solution (Lounis and Vanier, 2000), with $p$ indicating the type of distance-defining metric. Common values are $p=1$ (Manhattan, or Rectilinear distance), $p=2$ (Euclidean, or Cartesian), and $p=\infty$ (Tchebyschev). The $L_{p}$ solutions are important because they minimize distance to the ideal solution and generally lie in the "belly" of the front, precisely in the region where the model is most efficient. If the decision maker is zooming his analysis on this region, she may want to select an $L_{p}$ solution for implementation on the field.

If the Pareto front is known $L_{p}$-distance minimizing solutions can be derived by direct evaluation. In the case study the Pareto front is not a complete front but a subset thereof, thus solutions of Table 1 below may be slightly off the exact ones.
Table 1

Case study $L_{p}$ solutions.

\begin{tabular}{|c|c|c|c|c|}
\hline Solution & $O_{1}$ & $O_{2}$ & $\Delta L_{1}$ & $\Delta L_{2}$ \\
\hline$L_{1}$ & 58,600 & $1,855 \mathrm{k} €$ & $20.8 \%$ & $31.1 \%$ \\
\hline$L_{2}$ & 57,400 & $1,712 \mathrm{k} €$ & $23.4 \%$ & $28.7 \%$ \\
\hline$L_{\infty}$ & 56,000 & $1,563 \mathrm{k} €$ & $26.5 \%$ & $26.2 \%$ \\
\hline
\end{tabular}

In the table $\Delta L_{p}$ represents the relative distance to the ideal. Exact $L_{1}$ and $L_{\infty}$ distance minimizing solutions can be obtained without having to derive a complete front by using the technique of goal programming. See e.g. (Coutinho-Rodrigues et al., 2012B) for an example of how this was done in another bi-objective MILP problem.

\subsection{Discussion}

In order to see how the model and its solutions react to changes in parameters, costs and data, additional test runs were carried out, which are now discussed. These also help understanding the overall characteristics of the model and its scaling of computational time with problem size.

3.3.1 Comparison of repairs policies. Given its dominance over the partial/full recovery policy, it is always preferable to perform calculations in the model's native mixed recovery policy. Nevertheless, it is important to understand better how the Pareto fronts behave relative to each other.

Data, namely service levels and costs, provides a first hint on how the three recovery policies compare. This can be understood as follows. The strength of the mixed recovery policy stems from the fact it allows choosing either a partial or full repair for a particular system element, depending on what is more advantageous, i.e. on what has a better costbenefit ratio. If this ratio favors partial (full) repairs for a vast majority of elements, these repairs get chosen more often and the partial (full) recovery front tends to follow the mixed recovery front. When the ratio does not favor a particular type of repair, the best action is to mix the two types and consequently the mixed recovery front pulls away from the other two.

However, benefit bonuses have an impact as well. At low budgets the model tries to collect $B^{2}$ and $B^{R}$ bonuses, pushing solutions towards partial repairs. This drive is stronger when many initial service levels lie above the threshold $T$, as just a few partial repairs suffice to start reaching $B^{2}$ and $B^{R}$ bonus. This is what happened in the case study: despite data slightly favoring full repairs, the solutions profiles given in appendix B show that, for low budget, it is better instead to reach $B^{2}, B^{R}$-bonuses. Solutions profiles in that appendix confirm that indeed between 23,000 and 43,000 benefits the model assigns partial repairs to an extra 300 elements, opposite only 100 extra full repairs (Figure B1), allowing it to pick-up about $220 B^{2}$ and $B^{R}$ bonuses (Figure B2).

The preference for partial repairs at low budget, induced by $B^{2}$ and $B^{R}$, is the reason the partial and mixed recovery policies fronts sit close at these budget levels. Such behavior is expected in general, unless $B^{2}$ and $B^{R}$ are small or data is very skewed towards full repairs. High values of $B^{3}$ have comparatively little influence because they only start to impact when the budget is generous.

As budget goes up, the model starts to have more flexibility to select a repair type, and this is why the mixed 
recovery policy clearly outshines the other at lower midrange and higher budgets. However, it is hard to predict how all the effects (data, bonuses) combine. In appendix $\mathrm{C}$ a smaller case study of 23 sidewalks is presented, showing that $\mathrm{partial} / \mathrm{mix}$ recovery fronts can effectively detach at low budget. The smaller size of the problem also allowed to run the model with a benefit spacing of 10 units and the higher resolution of the results revealed that many solutions are unsupported, i.e. lie outside the convex hull of the Pareto front (Ehrgott, 2005).

3.3.2 Model parameters. The effect of bonuses values was discussed in the previous subsection. As to other model parameters, test runs with different values, i.e. weights, socio-economic importance, plateau limits and discount rates did not lead to any significant changes in the results.

The threshold parameter $T$ can however have a nontrivial effect on results. Since changing it modifies costs and benefits, it can alter cost-benefit ratio and favor one particular type of repair. A high $T$ can also increase computational complexity, as more possibilities open up for repairing system elements. In the case study test runs showed that high values $(T>\sim 0.75)$ tend to favor full repairs, as the cost differences between partial and full repairs decreased, whereas low values $(T<\sim 0.25)$ tend to favor partial repairs for the opposite reason. This effect was seen on the Pareto fronts of the test runs.

3.3.3 Calculation times. Because the model is designed for planning long-term actions, which usually have long inception times, it is conceivable that even very large problem instances can be solved using exact solvers.

Modern MILP solvers, such as CPLEX, allow the user to assign branch priorities, i.e. select which regions of the search space should be explored first. Test runs showed that computations speed up considerably when top priority is given to cost variables, then $B$-bonuses variables, and finally the individual $x_{i j}$. That $B$-bonuses variables should precede $x_{i j}$ is intuitive to understand, as each $B$-bonus impacts the values of various $x_{i j}$. All results of this article were derived with branch priorities set as above.

Note that the high value of $B^{3}$ in the case study mitigates the drive towards $B^{2}$ and $B^{R}$ bonuses at lower mid-range and higher budgets, leading to more variety in the choice of repairs and consequently a higher complexity of calculations. A test run with $B^{3}=0.50$ led to computation times of about half of those of the case study, owing to the lower complexity.

To get a grasp on complexity scalability for larger problems, a test run was carried out, with duplicated case study data. The cloned data was then randomly modified on initial service level, repair costs and socio-economic importance. The average run time of a mixed run rose from 33.4 seconds to 130 seconds, hinting at a square-law.

Should it, in any case, prove impossible to achieve exact results, it is always possible to implement heuristics to derive approximately non-dominated solutions. Good heuristic solutions are however not straightforward to derive because it is necessary to find an appropriate representation for the bonuses structure. The naïve approach of coding solutions into a chromosome of length $M \times N$ with binary/ternary values and running a standard evolutionary algorithm (e.g. NSGA II) will have difficulties in finding solutions with $B$-bonuses attributed, as that requires alignment of multiple individual variables.

Alternatively the decision maker may decide beforehand to implement an $L_{1}$ or $L_{\infty}$ solution. These solutions are attractive because they lie the region of high efficiency and can be derived in reasonable time, even for very large problem instances. Yet another way to shorten computation times is to derive a low resolution Pareto front, i.e. with large benefit spacing, look at it, and then do higher resolution runs on regions of the front the decision maker finds interesting.

\section{SUMMARY AND FUTURE WORK}

In this research a modeling approach for optimizing longterm repair actions on infrastructure systems with multiple elements was presented. The model helps deciding whether the individual system elements should be left untouched, partially or fully repaired, with an aim at maximizing benefits to its users and minimizing intervention costs. A distinctive feature of the model is that it captures the system/elements structure of the problem and possible (usually spatial) relations between the various systems, as well as their importance. It also takes into account volume discounts that can be expected for large-scale interventions. All these features and their combination makes the approach different than other repair models in the literature.

The model was applied to a real case study and compared to two simpler recovery policies, results showing that the model's peak efficiency lies in the region of lower midrange spending. The measure of the efficiency gains may vary, but is always present. The authors are strongly convinced that the model has a lot of scope for application in planning real life infrastructure maintenance/repair actions, enabling decision makers to get cost/benefit optimized solutions and analyze the trade-offs between these solutions.

An obvious extension of the model would be to have it cater for more than two repairs end-states and more than two cost discount plateaus. Another improvement would be to include different system types or to study the effects of setting a time horizon and doing a life-cycle analysis, reflecting eventual additional costs due to fast wear in the individual repair costs. The volume discount function, which was made linear to keep the model in MILP formulation (thus exactly solvable), can also be replaced by more realistic discount functions. The later may however lead to the appearance of non-linear terms in the formulation and thus force a heuristic solving approach. Such an approach, albeit approximate and non-trivial to implement, would also have the advantage of allowing for more flexibility in modeling discounts, including new discounts on the final combined price, should the whole work be auctioned off to a single contractor. The authors hope to address some of these issues in the near future. 


\section{ACKNOWLEDGEMENTS}

This work was partially supported by the Portuguese Foundation for Science and Technology under grant PEstOE/EEI/UI308/2014; and the initiative Energy for Sustainability of the University of Coimbra, supported by project Energy and Mobility for Sustainable Regions (EMSURE), under grant - CENTRO-07-0224-FEDER002004. The authors would also like to thank the civil engineering contractor 'Oliveiras, S.A.' and its CTO Tarcísio Fogeiro for helping with the case studies budgeting and technical field implementation issues.

\section{REFERENCES}

Adeli, H. \& Yu, G. (1995), An Integrated Computing Environment for Solution of Complex Engineering Problems Using the Object-Oriented Programming Paradigm and a Blackboard Architecture, Computers and Structures, 54(2), 255-265. DOI: 10.1016/00457949(94)00323-U

Adeli, H. \& Kao, W.-M. (1996), Object-Oriented Blackboard Models for Integrated Design of Steel Structures, Computers and Structures, 61(3), 545-561. DOI: 10.1016/0045-7949(96)00023-5

Adeli, H. \& Wu, M. (1998), Regularization Neural Network for Construction Cost Estimation, Journal of Construction Engineering and Management, ASCE, 124(1), 18-24. DOI: 10.1061/(ASCE)0733-9364(1998)124:1(18)

Adeli, H. \& Sarma, K. (2006), Cost Optimization of Structures - Fuzzy Logic, Genetic Algorithms, and Parallel Computing, John Wiley and Sons, West Sussex, United Kingdom. ISBN: 978-0-470-86733-4

Capuruço, R. \& Tighe, S. (2006), A Web-Centric Information Model for Managing Road Infrastructure Data, Computer-Aided Civil and Infrastructure Engineering 21(5), 357-368. DOI: 10.1111/j.1467-8667.2006.00442.x

Chuang, L. \& Adeli, H. (1993), Design-Independent CAD Window System Using the Object-Oriented Paradigm and HP X Widget Environment, Computers and Structures, 48(3), 433-440. DOI: 10.1016/0045-7949(93)90319-9

Cohon, J.L. (1978), Multiobjective Programming and Planning. Academic Press, New York. ISBN: 0121783502

Coutinho-Rodrigues, J., Tralhão, L. \& Alçada-Almeida, L. (2012A), Solving a Location-Routing Problem with a Multiobjective Approach: The Design of Urban Evacuation Plans. Journal of Transport Geography 22, 206-218. DOI: 10.1016/j.jtrangeo.2012.01.006

Coutinho-Rodrigues, J., Tralhão, L. \& Alçada-Almeida, L. (2012B), A bi-objective modeling approach applied to an urban semi-desirable facility location problem. European Journal of Operational Research, 223, 203-213. DOI: 10.1016/j.ejor.2012.05.037

Dimitrakos, T. \& Kyriakidis, E. (2007), An improved algorithm for the computation of the optimal repair/replacement policy under general repairs, European Journal of Operational Research 182(2), 775-782. DOI: 10.1016/j.ejor.2006.07.042

Durango, P. \& Madanat, S. (2002), Optimal maintenance and repair policies in infrastructure management under uncertain facility deterioration rates: an adaptive control approach, Transportation Research Part A: Policy and
Practice 36(9), $\quad 763-778 . \quad$ DOI: $10.1016 / \mathrm{S} 0965-$ 8564(01)00038-6

Ehrgott, M. (2005), Multicriteria Optimization, Springer, Berlin, Heidelberg. ISBN: 3540213988

Furuya, A. \& Madanat, S. (2013), Accounting for network effects in railway asset management. Journal of Transportation Engineering, 139(1), 92-100. DOI: 10.1061/(ASCE)TE.1943-5436.0000477

Gao, H. \& Zhang, X. (2013), A Markov-Based Road Maintenance Optimization Model Considering User Costs, Computer-Aided Civil and Infrastructure Engineering, 28(6), 451-464. DOI: 10.1111/mice.12009

Golabi, K., Kulkarni, R.B. \& Way, G.B. (1982), A Statewide Pavement Management System, Interfaces 12(6), 5-12. DOI: 10.1287/inte.12.6.5

González, A., Dueñas-Osorio, L., Sánchez-Silva, M. \& Medaglia, A. (2016), The Interdependent Network Design Problem for Optimal Infrastructure System Restoration, Computer-Aided Civil and Infrastructure Engineering, 31(5), 334-350. DOI: 10.1111/mice.12171

Halfawy, M., Newton, L. \& Vanier, D. (2005), Municipal Infrastructure Asset Management Systems: State-of-the-Art Review, National Research Council of Canada, Report No. 48339.

Hegazy, T., Elhakeem, A., Ahluwalia, S.S. \& Attalla, M. (2012), MOST-FIT: Support Techniques for Inspection and Life Cycle Optimization in Building Asset Management, Computer-Aided Civil and Infrastructure Engineering 27, 130-142. DOI: 10.1111/j.1467-8667.2011.00729.x

Jiang, X. \& Adeli, H. (2004), Object-Oriented Model for Freeway Work Zone Capacity and Queue Delay Estimation, Computer-Aided Civil and Infrastructure Engineering, 19(2), 144-156. DOI: 10.1111/j.14678667.2004.00344.X

Karim, A. \& Adeli, H. (1999), Object-Oriented Information Model for Construction Project Management, Journal of Construction Engineering and Management, ASCE, 125(5), 361-367. DOI: 10.1061/(ASCE)07339364(1999)125:5(361)

Karim, A. \& Adeli, H. (1999), CONSCOM: An OO Construction Scheduling and Change Management System, Journal of Construction Engineering and Management, $A S C E, \quad$ 125(5), 368-376. DOI: 10.1061/(ASCE)07339364(1999)125:5(368)

Kijima, M., Morimura, H. \& Suzuki, Y. (1988) Periodical replacement problem without assuming minimal repair, European Journal of Operational Research, 37(2), 194-203. DOI: 10.1016/0377-2217(88)90329-3

Kim, H. \& Adeli, H. (2001), Discrete Cost Optimization of Composite Floors using a Floating Point Genetic Algorithm, Engineering Optimization, 33(4), 485-501. DOI: $10.1080 / 03052150108940930$

Lee II, E., Mitchell, J. \& Wallace, W. (2007), Restoration of Services in Interdependent Infrastructure Systems: A Network Flows Approach. IEEE Transactions on Systems, Man and Cybernetics, Part C, 37(6), 1303-1317. DOI: 10.1109/TSMCC.2007.905859

Lounis Z. \& Vanier D. (2000), A Multiobjective and Stochastic System for Building Maintenance Management, Computer-Aided Civil and Infrastructure Engineering, 15(5), 320-329. DOI: 10.1111/0885-9507.00196 
Love, C., Zhang, Z., Zitron, M. \& Guo, R. (2000), A discrete semi-Markov decision model to determine the optimal repair/replacement policy under general repairs, European Journal of Operational Research 125(2), 398-409. DOI: 10.1016/S03772217(99)00009-0

Michele, D.S. \& Daniela, L. (2011), Decision-support tools for municipal infrastructure maintenance management, Procedia Computer Science 3, 36-41 DOI: 10.1016/B978-0-12-398364-0.00022-X

Natividade-Jesus, E., Coutinho-Rodrigues, J. \& Tralhao, L. (2013), Housing evaluation with web-SDSS in urban regeneration actions, Municipal Engineer, 166(3), 194-207. DOI: $10.1680 /$ muen.12.00022

Nurre, S., Cavdaroglu, B., Mitchell, J., Sharkey, T. \& Wallace, W. (2012), Restoring infrastructure systems: An integrated network design and scheduling (INDS) problem, European Journal of Operational Research, 223(3), 794806. DOI: $10.1016 /$ j.ejor.2012.07.010

Peng, F. \& Ouyang, Y. (2014), Optimal Clustering of Railroad Track Maintenance Jobs, Computer-Aided Civil and Infrastructure Engineering, 29(4), 235-247. DOI: 10.1111/mice. 12036

Pham, H., Suprasad, A. \& Misra, R.B. (1997), Availability and mean life time prediction of multistage degraded system with partial repairs, Reliability Engineering \& System Safety, 56(2), 169-173. DOI: 10.1016/S0951-8320(96)00140-8

Sarma, K. \& Adeli, H. (1998), Cost Optimization of Concrete Structures, Journal of Structural Engineering, ASCE, 124(5), 570-578. DOI: 10.1061/(ASCE)07339445(1998)124:5(570)

Sarma, K. \& Adeli, H. (2000), Cost Optimization of Steel Structures, Engineering Optimization, 32(6), 777-802. DOI: $10.1080 / 03052150008941321$

Sarma, K. \& Adeli, H. (2000), Fuzzy Discrete Multicriteria Cost Optimization of Steel Structures, Journal of Structural Engineering, ASCE, 126(11), 1339-1347. DOI: 10.1061/(ASCE)0733-9445(2000)126:11(1339)

Sarma, K. \& Adeli, H. (2001), Bi-Level Parallel Genetic Algorithms for Optimization of Large Steel Structures, Computer-Aided Civil and Infrastructure Engineering, 16(5), 295-304. DOI: 10.1111/0885-9507.00234

Tak, S., Kim, S. \& Yeo, H. (2013), Large-Scale Maintenance Optimization Problems for Civil Infrastructure Systems, in A.H. Gandomi, X. Yang, S. Talatahari and A.H. Alavi (eds.), Metaheuristic Applications in Structures and Infrastructures, Elsevier, pp. 519-538. DOI: 10.1016/B978-0-12-398364-0.00022-X

TRB - Transportation Research Board (2000). Highway Capacity Manual. Washington D.C.: National Academy of Sciences. ISBN 0-309-06681-6.

Wang, H. (2002), A survey of maintenance policies of deteriorating systems, European Journal of Operational Research, 139(3), 469-89. DOI: 10.1016/S03772217(01)00197-7

Wang, K.C.P. \& Zaniewski, J.P. (1996), 20/30 Hindsight: The New Pavement Optimization in the Arizona State Highway Network, Interfaces 26(3), 77-89. DOI: 10.1287/inte.26.3.77

Yu, G. \& Adeli, H. (1993), Object-Oriented Finite Element Analysis Using EER Model, Journal of Structural
Engineering, ASCE, 119(9), 2763-2781. DOI: 10.1061/(ASCE)0733-9445(1993)119:9(2763)

Zhang, X. \& Gao, H. (2012), Determining an Optimal Maintenance Period for Infrastructure Systems, ComputerAided Civil and Infrastructure Engineering, 27(7), 543554. DOI: $10.1111 / \mathrm{j} .1467-8667.2011 .00739 . x$

\section{APPENDIX A}

\section{Case study data - service levels and costs estimation}

Determination of initial and final service levels follows the methodology for evaluation of sidewalk performance presented in the Research Report of INESC-Coimbra (RR): http://www.uc.pt/en/org/inescc/res reports docs/RR 0420 15. Repair costs were estimated by Oliveiras S.A., a national civil engineering company (www.oliveiras.pt), who also suggested plateau lower limits and discount rates. Costs include wages and eventual demolitions necessary. Initial service levels, sidewalk length and HCM pedestrian density were surveyed by two civil engineers and averaged out.

Width: following Portuguese code of practice for sidewalk widths, service level was set to 0 for width lower than $0.90 \mathrm{~m}$ and to 1 for width greater than $3.0 \mathrm{~m}$, with linear interpolation for widths in-between. Costs for enlarging width to partial/full levels were estimated taking into account sidewalk extra pavement area needed (including base and subbase layers) and curb length.

Accessibilities and walking environment: following the $R R$, service level is measured in a 0-1 continuous scale and includes several factors for each element (accessibilities: crossings, access ramps, curb, vertical signaling; walk environment: vegetation, rest places, rubbish bins). Costs for partial/full repairs were calculated as the cheapest improvement that would make the service level reach the desired level. E.g. if an extra crossing or ramp would make the accessibility service level reach 0.65 , but the crossing were cheaper, that would be the partial repair cost.

The remaining elements have service levels reported in 0-4 Likert scales (see RR for details). These were normalized to 1 for model purposes, as requested by the approach. Owing to the discrete nature of Likert scales, some simplifying hypotheses had to be made to define service levels and calculate costs.

Pavement comfort: repair costs took into account type of substitute pavement and sidewalk area. A pavement type of comfort rated as borderline 3 was considered for partial repairs.

Conservation status: an average repair cost per squaremeter was estimated $(\mathrm{Cr})$ and total cost calculated as $\mathrm{Cr} \times$ sidewalk area $\times\left(Z-S_{i j}\right)$, with $Z=T$ for partial repairs and $Z=1$ for full repairs. In practice partial repairs can be interpreted as repairing the major cracks, stub toes, etc. and leaving minor issues behind. Full repairs means fixing all the problems.

Safety: service level takes into account traffic density and buffer zone type and width. Costs for reaching partial (full) levels were calculated as the cheapest way to improve the buffer zone to reach Likert safety level 3 (4).

Lightning: codes of practice suggest one lamp-post each $25 \mathrm{~m}$. An $S_{i j}=\mathrm{X}$ was considered to correspond to a density of X each $25 \mathrm{~m}$. The number of extra lamp-posts necessary 
to reach $65 \% / 100 \%$ of recommended density was calculated and transformed into costs.

Obstacles: an average cost per square-meter for removal of obstacles (such as misplaced trees or urban furniture) was estimated $(\mathrm{Co})$. Costs were estimated similarly to conservation status: $C o \times$ sidewalk area $\times\left(Z-S_{i j}\right)$.

\section{APPENDIX B}

\section{Case study solutions profiles}

The figures below summarize practical information on the case study mixed recovery policy solutions.

Figure B1 presents a count of individual element final service levels for each solution in the derived Pareto front. Sidewalks start out with 837 elements under $T, 702$ between $T$ and 1, and 293 fully operational elements (most of these are sidewalks over $3.0 \mathrm{~m}$ wide, which do not need enlargement, and sidewalks on quiet one-way and restricted streets, which have maximum safety). This initial situation provides 23,000 benefits to users. At 53,000 benefit, the minimum cost solution leaves 266 elements unrepaired at a final service below $T, 1040$ partially repaired, and 526 fully repaired. A complete overhaul would leave the 1832 elements fully repaired.

Figure B2 provides a count on the number of benefit bonuses awarded for each solution. Initially all sidewalks have at least one element below $T$, so, of the 23,000 benefits, none comes from $B$-bonuses. At 53,000 benefits, 153 sidewalks (of 229) end partially repaired in all their elements, 8 in fully repaired in all their elements, and 154 contiguity $\left(B^{R}\right)$ bonuses are achieved. At maximum benefits all the 229 sidewalks are fully repaired and 210 contiguity bonuses are achieved.

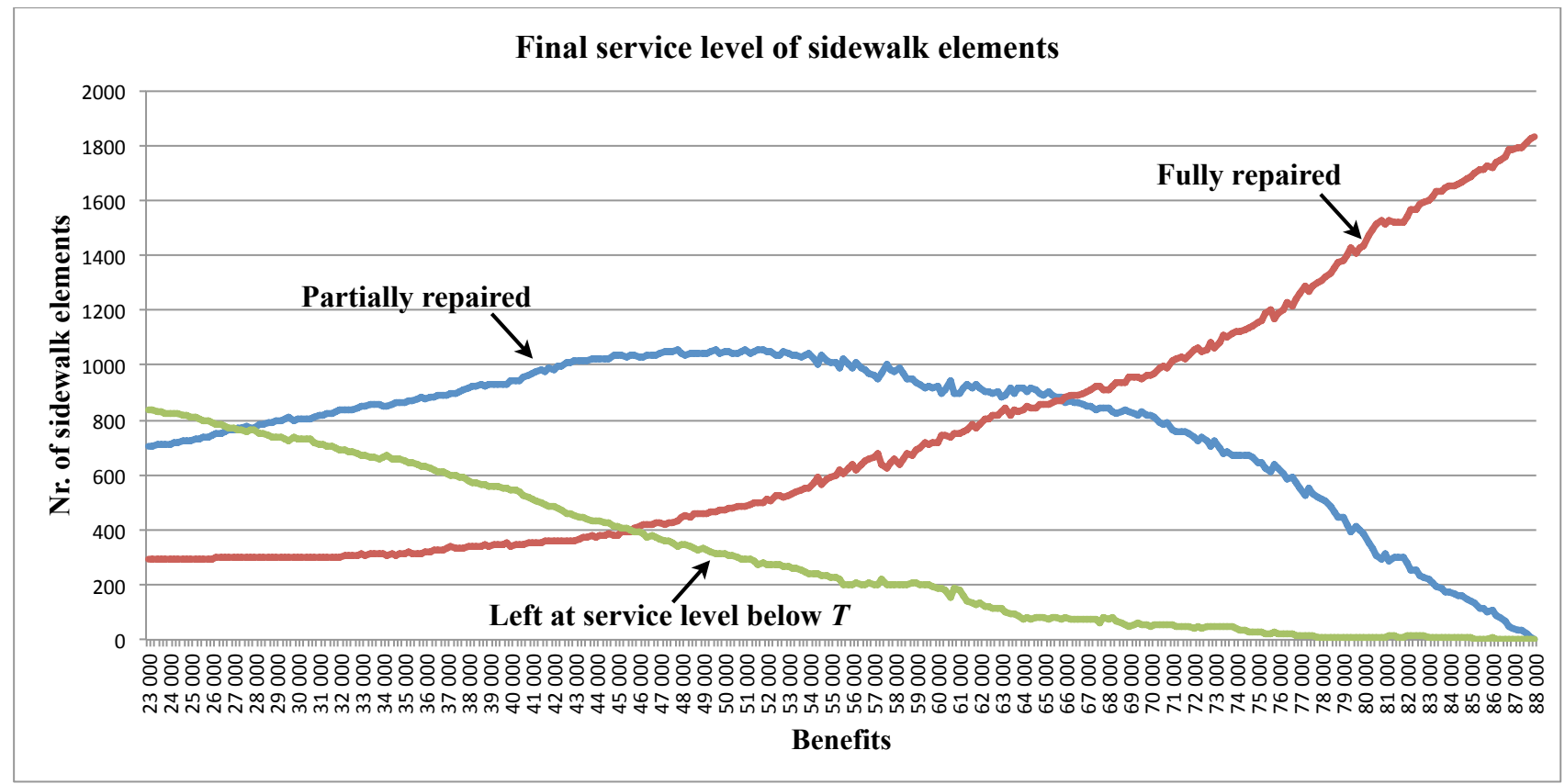

Figure B1 Sidewalk individual elements final service levels for Pareto solutions

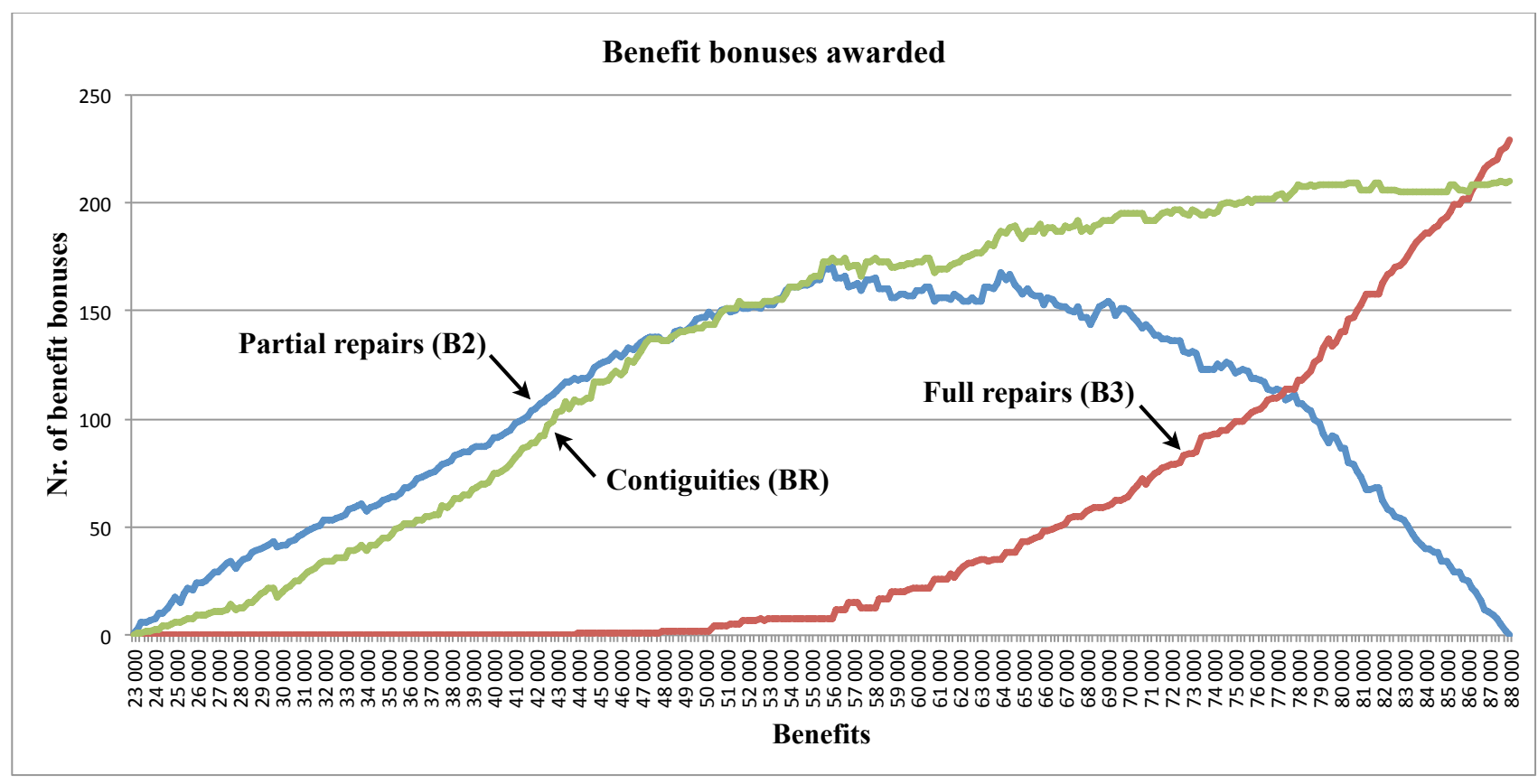


Figure B2 Benefit bonuses awarded for Pareto solutions

\section{APPENDIX C}

\section{Smaller case study of 23 sidewalks}

This case study features data from a subset of 23 sidewalks from the older neighborhood, the historical center of the city. Many of the sidewalks are located on streets belonging to a zone classified as World Heritage by UNESCO and have a lot of pedestrian traffic, mainly tourists.
Bonuses $B^{2}, B^{3}, B^{R}$ were set to $0.30 / 0.50 / 0.10$, and $T$ to 0.50 . Costs were recalculated for this $T$. The data features two strong pull effects on opposite directions: a very high cost-benefit skew towards full repairs and over $70 \%$ elements above $T$. The model was run for intervals of 10 benefit units and results for the three fronts are presented in Figure $\mathrm{C} 1$ below.

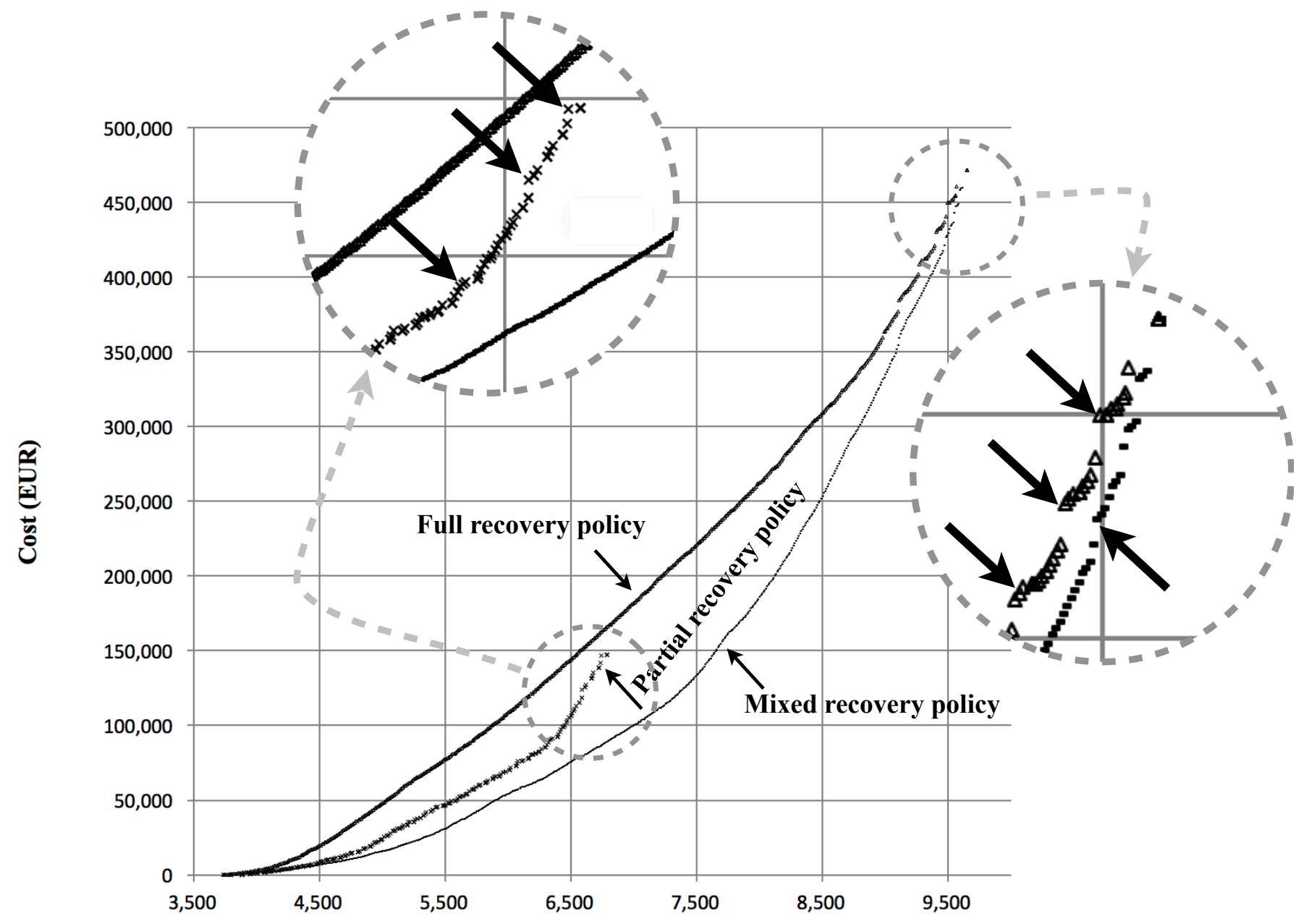

Benefits

Figure C1 Smaller case study of 23 sidewalks.

The two pull effects are evenly balanced, resulting in a detachment of all the three fronts from very early on. This detachment goes further way than in the main study, meaning that mixed recovery policy is more efficient in the smaller study, as compared to the alternative recovery policies.

Zooming on the higher resolution results clearly shows that many non-dominated solutions are unsupported. Some examples of these solutions are highlighted in the figure (arrows). Unsupported solutions cannot be expressed as convex combinations of the objectives and hence cannot be reached by common multiobjective methods such as weightedsum. A different method, such as the $\varepsilon$-constraint method used in this research, is required to find them. 